\title{
UBE2S promotes the proliferation and survival of human lung adenocarcinoma cells
}

\author{
Zhi Liu ${ }^{1,2} \&$ Lijun Xu ${ }^{1, *}$ \\ ${ }^{1}$ Department of Respiratory Medicine, The First Hospital of Jilin University, Changchun 130021, ${ }^{2}$ Affiliated Hospital of Inner Mongolia \\ University for the Nationalities, Tongliao 028000, China
}

\begin{abstract}
Ubiquitin-conjugating enzyme E2S (UBE2S), a family of E2 protein in the ubiquitination process, is involved in development of various cancers. However, its role in lung adenocarcinoma, has not been well elucidated. In this report, we attempted to investigate expression and function of UBE2S in lung adenocarcinoma. Up-regulation of UBE2S at mRNA, and protein level, was observed in human cancer tissues and lung adenocarcinoma cells. Higher UBE2S expression correlated with poorer prognosis of lung adenocarcinoma patients. UBE2S expression was efficiently suppressed by lentivirus-mediated shRNA strategy in A549 cells, and UBE2S silencing led to reduced cell proliferation, colony formation, and enhanced apoptosis. Inverse results were observed, in UBE2S over-expressed H1299 cells. Microarray analysis indicated that a large number of genes were regulated by UBE2S, and p53 signaling pathway may be critical, to the role of UBE2S in cancer development. Together, UBE2S could be a potential target for lung adenocarcinoma. [BMB Reports 2018; 51(12): 642-647]
\end{abstract}

\section{INTRODUCTION}

Lung cancer is the most common cause of cancer-related death in men, and second most common cancer in women, after breast cancer (1). The two main types of lung cancers are small-cell lung carcinoma (SCLC), and non-small-cell lung carcinoma (NSCLC). Lung adenocarcinoma is one of the most common subtypes of NSCLC, accounting for approximately $40 \%$ of all lung cancers $(2,3)$. In recent years, various therapeutic strategies were increasingly raised, and used for lung adenocarcinoma, but its five-year overall survival (OS)

*Corresponding author. Tel: +86-0431-88782319; Fax: +86-043188782320; E-mail: lijunxu001@sina.com

https://doi.org/10.5483/BMBRep.2018.51.12.138

Received 4 July 2018, Revised 11 August 2018, Accepted 23 October 2018

Keywords: Apoptosis, Lung adenocarcinoma, P53, Proliferation, UBE2S remains very low (4). Thus, there is a need to explore novel and effective therapeutic targets for lung adenocarcinoma.

Ubiquitination could affect proteins in many ways, mainly involved in activation, conjugation and the ligation process, mediated by ubiquitin-activating enzymes (E1s), ubiquitinconjugating enzymes (E2s), and ubiquitin ligases (E3s), respectively. Ubiquitin-conjugating enzyme E2S (UBE2S) is a family of E2 proteins, in the ubiquitination process. Recently, it has been shown that UBE2S plays a vital role in regulating DNA damage-induced transcriptional silencing, through catalyzing Lys11-linkage ubiquitination (5-8). Previous studies showed that aberrant expression of UBE2S was observed, in various cancers including breast, esophageal, cervical and renal cancers (9-14). Also, a previous study indicated that UBE2S played an important role in determining malignancy properties of human colorectal cancers, and promoted its development (15). However, the critical function of UBE2S in lung adenocarcinoma, remains largely unclear.

In this report, we investigated expression and function of UBE2S in lung adenocarcinoma. UBE2S was up-regulated in human lung cancer tissues, and lung adenocarcinoma cells A549. Patients with higher expression of UBE2S had poorer prognosis, compared with patients with lower UBE2S expression. UBE2S silencing suppressed proliferation and colony formation, and induced the apoptosis of lung cancer cells. Also, microarray assay showed that numerous genes were regulated by UBE2S. p53 signaling pathway may be essential to the function of UBE2S in lung cancer. Thus, UBE2S may be a potential therapeutic target in lung adenocarcinoma.

\section{RESULTS}

UBE2S expression in lung cancer tissues and cells

We first investigated protein abundance of UBE2S in lung cancer samples, using immuno-histochemical assay. Clinical and pathological characteristics of patients were described in Table S1. Initially, UBE2S was moderately up-regulated in cancer tissues, comparing with normal tissues (Fig. 1A and Table S2). Expression of UBE2S was correlated with clinical T phase of lung cancer patients (Table S1) in our data. Then mRNA expression of UBE2S in lung cancer and normal tissues was analyzed, based on the TCGA database. Results showed 


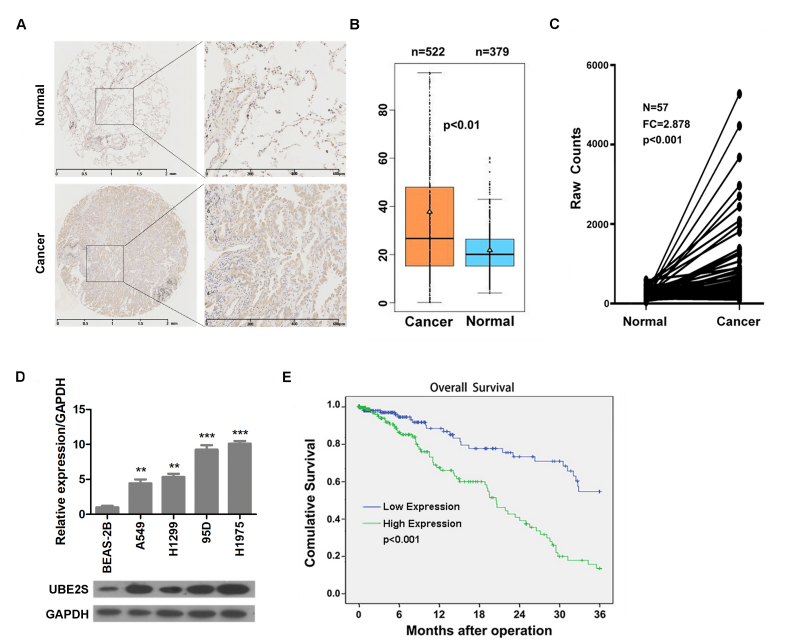

Fig. 1. UBE2S expression is increased in lung cancer tissues and cells and high expression of UBE2S predicts poor outcome of lung cancer patients. (A) Immuno-histochemical analysis of UBE2S expression in lung cancer and normal tissues. (B) UBE2S mRNA expression in lung cancer $(\mathrm{n}=522)$ and normal tissues $(\mathrm{n}=$ 379) derived from the Cancer Genome Atlas (TCGA). $P<0.01$. (C) UBE2S mRNA expression in lung cancer and adjacent normal tissues derived from the Cancer Genome Atlas (TCGA). FC, Fold Change, $\mathrm{n}=57, \mathrm{P}<0.001$. (D) UBE2S mRNA expression and protein level were determined respectively by qRT-PCR and Western blot assays in BEAS-2B, 95D, H1299, H1975 and A549 cells. ${ }^{* * P}<0.01,{ }^{* * * P}<0.001$. (E) Lung cancer patients were divided into UBES2 high (Green line) and low (Blue line) expression groups. Overall survival of patients was determined after operation. $\mathrm{P}<0.001$.

that UBE2S mRNA level increased in lung cancer tissues, as compared with non-paired or paired normal tissues (Fig. 1B and C). Interestingly, UBE2S mRNA expression correlated with clinical $\mathrm{N}$ migration, and pathological stages of lung cancer patients in TCGA data (Table S3). Also, UBE2S mRNA and protein expression were higher in lung cancer cells 95D, $\mathrm{H} 1299, \mathrm{H} 1975$ and lung adenocarcinoma cells A549, than in BEAS-2B normal cells (Fig. 1D). Importantly, patients with higher UBE2S expression exhibited shorter overall survival, than those with lower ones (Fig. 1E). We predict that UBE2S is involved in lung cancer development.

\section{UBE2S knockdown suppresses the proliferation and growth of A549 cells}

To evaluate the effect of UBE2S on cell proliferation, lentivirus-mediated UBE2S knockdown and over-expression were performed in A549 cells and H1299 cells, respectively. qRT-PCR and Western blot results showed that UBE2S was efficiently silenced in shUBE2S-1 and shUBE2S-2 A549 cells (Fig. 2A and B), and it was over-expressed in H1299 cells (Fig. 2C and D). Then shCtrl and shUBE2S A549 cells were subjected to cell proliferation analysis. Cell growth screening assay showed that UBE2S knockdown inhibited proliferation
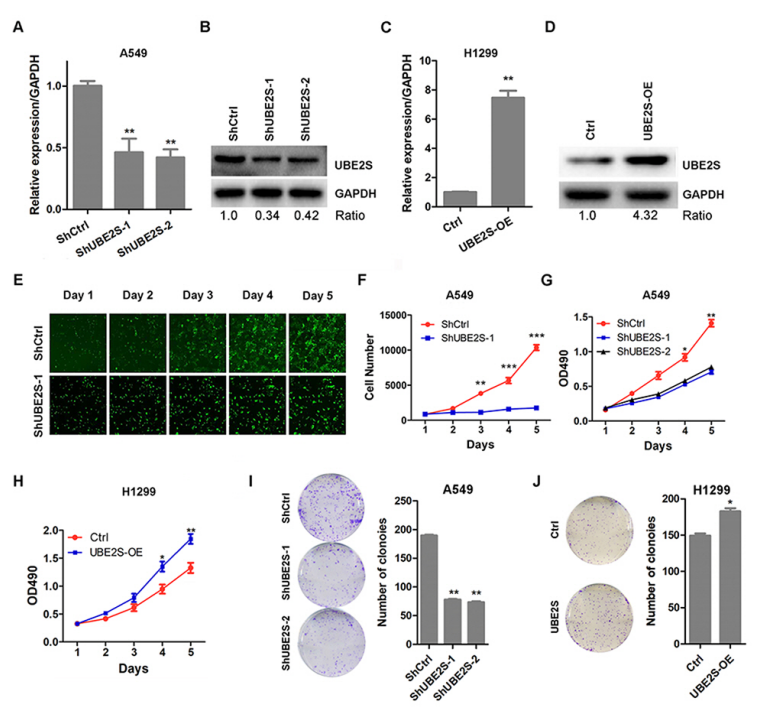

Fig. 2. UBE2S knockdown suppresses the proliferation of $A 549$ cells. (A, B) shCtrl, shUBE2S-1 and shUBE2S-2 A549 cells were subjected to qRT-PCR (A) and Western blot (B) analysis of UBE2S. GAPDH serve as internal control. **P $<0.01$. (C, D) Control (Ctrl) and UBE2S over-expressed (UBE2S-OE) H1299 cells were subjected to qRT-PCR (C) and Western blot (D) analysis of UBE2S. GAPDH serve as internal control. $* * P<0.01$. (E, F) (E) Representative images of high-content screening (HCS) of shCtrl (top) and shUBE2S-1 (bottom) A549 cells from day 1 to day 5 . HCS detected the numbers of viable cells. (F) Relative quantification of $\mathrm{HCS}$ results (Cell count). $* * \mathrm{P}<0.01, * * * \mathrm{P}<$ 0.001. (G) Viability of shCtrl, shUBE2S-1 and shUBE2S-2 A549 cells was determined by MTT assay. $* P<0.05, * * P<0.01$. (H) Viability of Control (Ctrl) and UBE2S over-expressed (UBE2S-OE) H1299 cells was determined by MTT assay. ${ }^{*} \mathrm{P}<$ $0.05, * * P<0.01$. (I) shCtrl, shUBE2S-1 and shUBE2S-2 A549 cells were subjected to colony formation assay. ${ }^{*} \mathrm{P}<0.01$. (J) Control (Ctrl) and UBE2S over-expressed (UBE2S-OE) H1299 cells were subjected to colony formation assay. ${ }^{*} \mathrm{P}<0.05$.

of A549 cells (Fig. 2E and F). Consistently, MTT analysis also indicated that the viability of A549 cells was suppressed by shUBE2S-1 and shUBE2S-2 (Fig. 2G). Too, colony number was reduced in shUBE2S-1 and shUBE2S-2 A549 cells as compared with shCtrl cells (Fig. 2l). By contrast, UBE2S over-expression increased viability and colony formation capacity of H1299 cells (Fig. 2H and J). Collectively, UBE2S promotes proliferation and colony formation of A549 cells.

\section{UBE2S enhances the BrdU incorporation and suppresses the apoptosis of lung cancer cells}

Next, BrdU incorporation assay was performed, to verify the effect of UBE2S on cell proliferation. Consistent with Fig. 2, BrdU incorporation was reduced in shUBE2S-1 and shUBE2S-2 A549 cells, and was enhanced in UBE2S over-expressed $\mathrm{H} 1299$ cells (Fig. 3A and B). To determine the role of UBE2S on cell survival, shCtrl and shUBE2S A549 cells were subjected to apoptosis analysis through flow cytometry. 


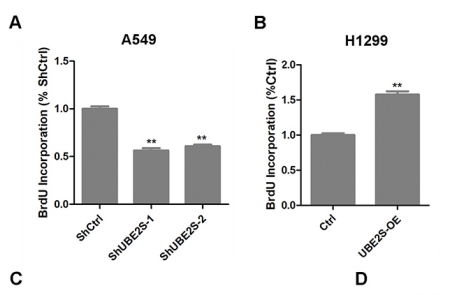

c

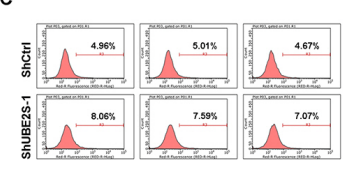

E
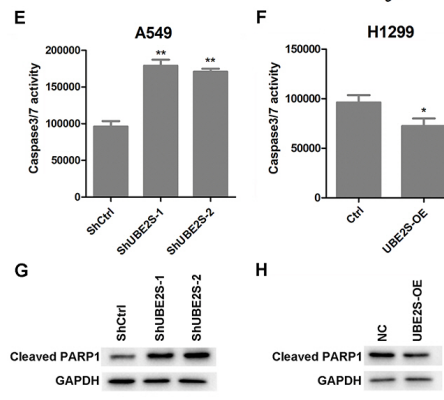

Fig. 3. UBE2S induces the BrdU incorporation and suppresses apoptosis of lung cancer cells. (A) The BrdU incorporation of shCtrl, shUBE2S-1 and shUBE2S-2 A549 cells. $* * P<0.01$. (B) The BrdU incorporation of Control (Ctrl) and UBE2S overexpressed (UBE2S-OE) H1299 cells. $* * P<0.01 .(C, D)$ The apoptosis of shCtrl and shUBE2S-1 A549 cells was determined by Flow cytometry. (C) Representative images of the flow cytometry results, and (D) quantification of apoptosis. $* * P<0.01$. (E) Caspase 3/7 activity of shCtrl, shUBE2S-1 and shUBE2S-2 A549 cells. ${ }^{*} \mathrm{P}<0.01$. (F) Caspase $3 / 7$ activity of Ctrl and UBE2S-OE H1299 cells. $* P<0.05$. (G) Western blot analysis of cleavedPURP1 in shCtrl, shUBE2S-1 and shUBE2S-2 A549 cells. (H) Western blot analysis of cleaved-PURP1 in Ctrl and UBE2S-OE H1299 cells.

We observed that the number of cell apoptosis increased in shUBE2S A549 cells, as compared to shCtrl cells (Fig. 3C and D). Additionally, UBE2S silencing mediated by shUBE2S-1 and shUBE2S-2, resulted in increased caspase 3/7 activity and UBE2S ectopic expression, led to decreased caspase 3/7 activity (Fig. 3E and F). Western blot analysis showed that cleaved PARP1 was up-regulated in shUBE2S-1 and shUBE2S-2 A549 cells, and was down-regulated in UBE2S over-expressed $\mathrm{H} 1299$ cells (Fig. 3G and H). Together, UBE2S promoted cell proliferation and suppressed apoptosis in lung cancer cells.

\section{Profiling the genes regulated by UBE2S}

To explore downstream genes and pathways regulated by UBE2S, we performed microarray analysis to identify differential gene expression in shCtrl and shUBE2S A549 cells. A total of 171 genes were up-regulated, and 287 genes were down-regulated by UBE2S knockdown (Fig. 4A). Pathway enrichment analysis showed that UBE2S-regulated genes were
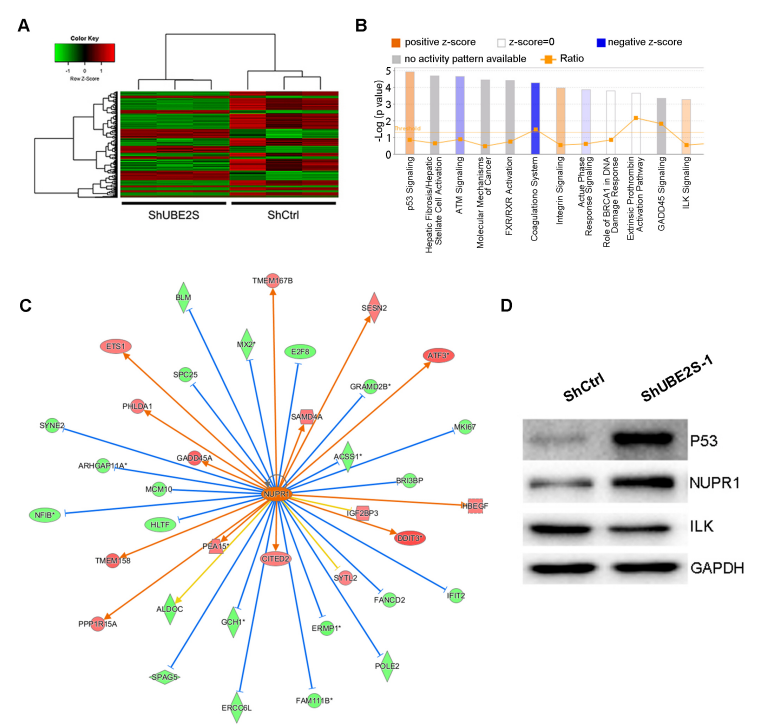

Fig. 4. Dysregulated genes in A549 cells after UBE2S knockdown. (A) A total of 458 genes were regulated (171 genes up-regulated and 287 genes down-regulated) by UBE2S knockdown in A549 cells ( $\mathrm{P}<0.05$, fold change $>1.5$ ). (B) Pathway enrichment was analyzed by IPA software. Orange, activated pathway. Blue, suppressed pathway. (C) Correlation map of up-regulated NUPR1 and its potential downstream targets. Red and orange box, up-regulated gene. Green box, down-regulated gene. Blue arrow, consistent repression. Orange arrow, consistent activation. Yellow arrow, inconsistent correlation. (D) Western blot analysis of P53, NUPR1 and ILK in shCtrl and shUBE2S A549 cells.

mainly involved in activation of P53, Integrin and ILK signaling pathway, and suppression of Coagulation, ATM and Acute Phase Response signaling pathway (Fig. 4B). We also performed correlation analysis of downstream targets regulated by UBE2S. As shown in Fig. 4C, the green boxes represented down-regulated genes. Red ones represented up-regulated genes. Previous studies have shown that these dysregulated genes were also regulated by NUPR1. Correlation analysis indicated that NUPR1 served as mediator between downstream targets and UBE2S (Fig. 4C). This suggested that UBE2S knockdown may cause up-regulation of NUPR1, which correlated with the dysregulation of various genes. Thus, NUPR1 maybe an important downstream target for UBE2S. Also, consistent with microarray data, P53 and NUPR1 were up-regulated, and ILK was down-regulated by UBE2S knockdown in A549 cells (Fig. 4D). Together, UBE2S regulation of these genes maybe important, for its role in lung cancer.

\section{DISCUSSION}

In the last decade, treatment strategies targeting specific molecular subsets of NSCLC, especially lung adenocarcinoma, have acquired remarkable success. Typically, small molecule tyrosine kinase inhibitors of epidermal growth factor (EGFR), 
and anaplastic lymphoma kinase (ALK), provided a comparable degree of clinical benefit to patients with mutations in EGFR and rearrangements involving the ALK gene (16-22). However, the function of these targeted agents remains limited, partly due to primary or secondary resistance. These facts facilitated us to explore molecular mechanisms underlying progression of lung adenocarcinoma, thus to identify potential targets for developing effective targeted treatment.

UBE2S, a family of E2 proteins, mainly contributes to poly-ubiquitination or ubiquitin chain formation during the ubiquitination process (23-25). It plays an essential role in timely degrading protein regulators of the cell cycle, for completion of cell division (26). Up-regulation of UBE2S was observed in various cancers. Previous studies have reported that UBE2S participates in progression of human cancer. For example, UBE2S promotes colorectal cancer development, and hepatocellular carcinoma cell proliferation and migration $(15,27)$. Reduction of UBE2S is also involved in dietary flavonoids, luteolin, and quercetin inhibition of cervical cancer invasion (28). In this study, we found that UBE2S up-regulated in lung cancer tissues and several cell lines, and UBE2S expression inversely correlated with the prognosis of lung adenocarcinoma patients. This suggested that UBE2S may contribute to development of lung adenocarcinoma. We subsequently knocked down UBE2S using lentivirus strategy, and found that UBE2S silencing inhibited proliferation and colony formation of A549 cells and induced its apoptosis. Results suggested that UBE2S was a potential oncogene for lung adenocarcinoma.

Functional and mechanistic studies have identified various downstream targets of UBE2S in cancers. In hepatocellular carcinoma, UBE2S potentiates the ubiquitination of p53, and cancer cell proliferation and migration (27). p21, a downstream effecter of p53, is also regulated by UBE2S in oral squamous cell carcinoma (29). In addition, UBE2S promotes colorectal cancers through stabilizing $\beta$-Catenin via K11-linked polyubiquitination (15). Here, using microarray assay, we showed that UBE2S knockdown led to dysregulation of various genes. Pathway enrichment suggested that p53 signaling pathway, was negatively regulated by UBE2S. Additionally, UBE2S knockdown activated integrin and ILK signaling, and inhibited the coagulation system. Still, further studies should be performed, to illustrate participation of these signaling pathways in lung cancer development.

In summary, our study showed that UBE2S was a potential oncogene in lung adenocarcinoma.UBE2S was up-regulated in human lung cancer tissues and cell lines. Knockdown of UBE2S significantly inhibited proliferation and colony formation of lung adenocarcinoma cell A549, and induced its apoptosis. Microarray analysis revealed that UBE2S could regulate expression of different genes, including p53 signaling pathway. Thus, targeting UBE2S is a potential targeted strategy for lung adenocarcinoma.

\section{MATERIALS AND METHODS}

\section{Microarray of human lung adenocarcinoma and adjacent normal samples}

HLug-Ade060PG-01 microarrays of lung adenocarcinoma, and adjacent normal samples were obtained from Outdo Biotech. This array contains a total of 30 samples with 5 stage I, 15 stage II, 10 stage III lung adenocarcinoma samples, and 30 adjacent normal samples. A core represented a separate case, and each sample was fixed in formalin. 5 - $\mu$ m-thick slices coated by paraffin, were subjected to immunohistochemistry staining of UBE2S.

\section{TCGA gene expression data}

UBE2S transcriptome expression datasets and clinical information, were downloaded from The Cancer Genome Atlas (http://cancergenome.nih.gov). Five-hundred twenty-two tumor tissues, 379 normal tissues, and 57 pairs of cancer and adjacent normal tissues were used, for analyzing UBE2S expression in this study.

\section{Cell culture}

Lung epithelial cells BEAS-2B derived from bronchial epithelium of one normal subject, were obtained from the American Type Culture Collection. Lung adenocarcinoma cells 95D, H1299, H1975 and A549, were purchased from Cell Bank of the Chinese Academy of Sciences (Shanghai, China). All cells were cultured in RPMl1640 medium containing 10\% fetal bovine serum, and $1 \%$ antibiotics. These cells were maintained at $37^{\circ} \mathrm{C}$ incubator containing $5 \% \mathrm{CO}_{2}$.

\section{UBE2S knockdown assay}

UBE2S was knocked down using pGCSIL-GFP lentivirus vectors in A549 cells. pGCSIL-GFP was co-transfected with pHelper1.0 and Helper2.0 into 293T cells using Lipofectamine TM 2000 (Invitrogen, Shanghai, China). Viral supernatants were collected 48 hours later, and filtered through $0.45 \mu \mathrm{m}$ filters. Then, supernatants were used to infect A549 cells. Targeted sequences were as follow: shCtrl, 5'-TTCTCC GAACGTGTCACGT-3'; shUBE2S-1, 5'-CATATGCTGGAGGTC TGTT-3'; shUBE2S-2, 5'-GGGCTCTCTTCCTCCTTCCAC-3'. qRT-PCR and Western blot were used to determine infection efficacy.

\section{UBE2S over-expression in $\mathrm{H} 1299$ cells}

The cDNA sequence of UBE2S was inserted into the $\mathrm{pCDH}$ lentivirus vector. Ctrl or UBE2S pCDH vectors, with PSPAX2 and PDM2G packaging vectors, were transfected into 293T cells. Three days later, virus supernatants were collected, filtered through $0.45 \mu \mathrm{m}$ filters, and then subjected to infecting H1299 cells. UBE2S over-expression was detected by qRT-PCR and Western blot assays.

\section{Westem blot}

A549 cells expressing shCtrl or shUBE2S lentivirus were 
cultured for 48 hours and used for protein isolation. In brief, culture medium was removed and cells were washed by PBS. Lysis buffer $(100 \mathrm{mM}$ Tris- $\mathrm{HCl}, \mathrm{pH}=7.4 \mid 0.15 \mathrm{M} \mathrm{NaCl} ; 5$ mM EDTA, $\mathrm{pH}=8.0 ; 1 \%$ Triton X100; $5 \mathrm{mM}$ DTT; $0.1 \mathrm{mM}$ PMSF) was added into $6 \mathrm{~cm}$ plates to extract total protein. BCA Protein Assay Kit (Pierce, Rockford, IL, USA) was used for protein quantification. A total of $30 \mu \mathrm{g}$ protein lysis were loaded into SDS-PAGE electrophoresis, and subsequently transferred onto PVDF transmembrane. Membranes were blocked with $5 \%$ skim milk for 1 hour at room temperature, and then incubated with primary antibodies overnight at $4^{\circ} \mathrm{C}$. After washing membranes with PBST, HRP conjugated secondary antibodies were added. ECL-Plus kit (Amersham Biosciences, Pollards Wood, UK) was used to detect immunoactivity. Primary antibody against UBE2S (ab197945), p53 (ab1431) and NUPR1 (ab6028) were purchased from Abcam. Primary antibody against ILK (\#3856) and Cleaved PARP1 (\#5625) were purchased from Cell Signaling Technology. GAPDH primary antibody (SC-32233) and secondary antibodies were obtain from Santa Cruz.

\section{Total RNA isolation and quantitative real-time PCR}

shCtrl and shUBE2S A549 cells were cultured for 48 hours, and washed by PBS. Trizol reagent (Invitrogen) was added into wells, and total RNA was isolated using RNeasy Mini kit (QIAGEN), according to manufacturer's instructions. A total of $1 \mu \mathrm{g}$ RNA was subjected to reverse-transcription using Rever Tra Ace ${ }^{\circledR}$ qPCR RT Master Mix with gDNA Remover (TOYOBO). TransStart Top Green qPCR SuperMix (TransGen Biotech) was used for quantitative real-time PCR on an IQ-5 machine. The primer sequences are as follow:UBE2Sforward, 5'-GTGCTCAAGAGGGACTGGACG-3', and reverse, 5'-GCAG ACTCGGGGTTAGGGTG-3'; GAPDH forward, 5'-TGACTTCA ACAGCGACACCCA-3', and reverse, 5'-CACCCTGTTGCTG TAGCCAAA-3'. GAPDH serves as internal control.

\section{High-content screening assay}

High-content screening (HCS) assay was used to detect the number of viable cells. This system is a computerized, automated fluorescence-imaging microscope which automatically detects stained cells, and analyzes intensity and distribution of fluorescence in each cell. Briefly, shCtrl and shUBE2S A549 cells were cultured in 96-well plates for 5 days. Cell numbers were scanned by a fluorescence-imaging microscope of $20 \times$ objective using ArrayScan $^{\mathrm{TM}}$ HCS software (Cellomics Inc). Intensity and distribution of green fluorescence indicated viable cells.

\section{MTT assay}

shCtrl and shUBE2S A549 cells were seeded in 96-well plates at a density of 3000 cells per well. 1, 2, 3, 4 and 5 days later, each well was washed by PBS, and then 3-(4,5-dimethyl2-yl)-2,5-diphenyltetrazolium bromide (MTT) solution (5 mg/ml) was added into the wells. Three hours later, MTT solution was removed, and dimethyl sulfoxide (DMSO) was added. Ten minutes later, cell viability was determined by detecting OD value at $490 \mathrm{~nm}$ on a micro-plate reader.

\section{Colony formation assay}

Equal number of shCtrl and shUBE2S A549 cells (800 cells per well) were seeded in six-well plates. After cultured for 12 days, cell colonies were fixed by methanol for half an hour, and stained with Giemsa solution for 15 minutes. The colony number was analyzed by a fluorescence microscopy (Olympus).

\section{BrdU incorporation assay}

BrdU incorporation assay was performed to determine DNA synthesis in proliferating cells using a BrdU kit (Roche, No.11647229001), following manufacturer's instructions.

\section{Apoptosis analysis}

Apoptosis of shCtrl and shUBE2S A549 cells detected by annexin V-APC kit (Ebioscience, USA), following manufacturer's instructions. Cells were washed by PBS, and re-suspended by staining buffer. After adding $5 \mu \mathrm{l}$ annexin V-APC into $100 \mu \mathrm{l}$ cell suspension, the mixture was incubated for 15 minutes at room temperature. Then they were subjected to flow cytometry detection of cell apoptosis (FACSCalibur, Becton-Dickinson, USA).

\section{Caspase $3 / 7$ activity analysis}

A total of 10,000 cells were seeded into each well of the 96-well plates, and $100 \mu$ l Caspase-Glo reagent (Promega) was added into each well. Then plates were rotated at 300-500 rpm for 30 minutes, and incubated at room temperature for 90 minutes activity was detected by the microplate reader.

Gene expression profiling by microarray assay in A549 cells RNA was extracted from shCtrl and shUBE2S A549 cells using Trizol reagents. Genes were expressed differentially when meeting criterion of fold change $>2$ and $P$ value $<0.05$. Pathway enrichment or gene network analysis was performed, using Ingenuity Pathway Analysis (IPA). Affymetrix human GeneChip primeview was used for gene expression analysis, according to protocols as described previously (30).

\section{Statistical analysis}

GraphPad prism 6.0 was used to analyze data as shown by mean \pm SEM of at least three independent repeats. All results were based on three independent experiments. Difference between the two groups was subjected to unpaired students' $t$ tests. Difference among the groups was analyzed by one-way ANOVA. $P$ value less than 0.05 was statistical significance.

\section{CONFLICTS OF INTEREST}

The authors have no conflicting interests. 


\section{REFERENCES}

1. Jemal A, Thomas A, Murray T and Thun M (2002) Cancer statistics, 2002. CA Cancer J Clin 52, 23-47

2. Imielinski M, Berger AH, Hammerman PS et al (2012) Mapping the hallmarks of lung adenocarcinoma with massively parallel sequencing. Cell 150, 1107-1120

3. McGuire S (2016) World Cancer Report 2014. Geneva, Switzerland: World Health Organization, International Agency for Research on Cancer, WHO Press, 2015. Adv Nutr 7, 418-419

4. Alberg AJ, Brock MV, Ford JG, Samet JM and Spivack SD (2013) Epidemiology of lung cancer: Diagnosis and management of lung cancer, 3rd ed: American College of Chest Physicians evidence-based clinical practice guidelines. Chest 143, e1S-e29S

5. Paul A and Wang B (2017) RNF8- and Ube2S-Dependent Ubiquitin Lysine 11-Linkage Modification in Response to DNA Damage. Mol Cell 66, 458-472.e455

6. Wu T, Merbl Y, Huo Y, Gallop JL, Tzur A and Kirschner MW (2010) UBE2S drives elongation of K11-linked ubiquitin chains by the anaphase-promoting complex. Proc Natl Acad Sci U S A 107, 1355-1360

7. Garnett MJ, Mansfeld J, Godwin C et al (2009) UBE2S elongates ubiquitin chains on APC/C substrates to promote mitotic exit. Nat Cell Biol 11, 1363-1369

8. Bremm A, Freund SM and Komander D (2010) Lys11-linked ubiquitin chains adopt compact conformations and are preferentially hydrolyzed by the deubiquitinase Cezanne. Nat Struct Mol Biol 17, 939-947

9. Penas C, Ramachandran V and Ayad NG (2011) The APC/C Ubiquitin Ligase: From Cell Biology to Tumorigenesis. Front Oncol 1, 60

10. Chen MF, Lee KD, Lu MS et al (2009) The predictive role of E2-EPF ubiquitin carrier protein in esophageal squamous cell carcinoma. J Mol Med (Berl) 87, 307-320

11. Roos FC, Evans AJ, Brenner W et al (2011) Deregulation of E2-EPF ubiquitin carrier protein in papillary renal cell carcinoma. Am J Pathol 178, 853-860

12. Liang J, Nishi H, Bian ML et al (2012) The ubiquitinconjugating enzyme E2-EPF is overexpressed in cervical cancer and associates with tumor growth. Oncol Rep 28, 1519-1525

13. Ayesha AK, Hyodo T, Asano E et al (2016) UBE2S is associated with malignant characteristics of breast cancer cells. Tumour Biol 37, 763-772

14. Jung CR, Hwang KS, Yoo J et al (2006) E2-EPF UCP targets pVHL for degradation and associates with tumor growth and metastasis. Nat Med 12, 809-816

15. Li Z, Wang Y, Li Y et al (2018) Ube2s stabilizes beta-Catenin through K11-linked polyubiquitination to promote mesendoderm specification and colorectal cancer development. Cell Death Dis 9, 456

16. Maemondo M, Inoue A, Kobayashi K et al (2010) Gefitinib or chemotherapy for non-small-cell lung cancer with mutated EGFR. N Engl J Med 362, 2380-2388

17. Rosell R, Carcereny E, Gervais R et al (2012) Erlotinib versus standard chemotherapy as first-line treatment for
European patients with advanced EGFR mutation-positive non-small-cell lung cancer (EURTAC): a multicentre, open-label, randomised phase 3 trial. Lancet Oncol 13, 239-246

18. Zhou C, Wu YL, Chen G et al (2011) Erlotinib versus chemotherapy as first-line treatment for patients with advanced EGFR mutation-positive non-small-cell lung cancer (OPTIMAL, CTONG-0802): a multicentre, openlabel, randomised, phase 3 study. Lancet Oncol 12, 735-742

19. Mitsudomi T, Morita S, Yatabe $Y$ et al (2010) Gefitinib versus cisplatin plus docetaxel in patients with non-small-cell lung cancer harbouring mutations of the epidermal growth factor receptor (WJTOG3405): an open label, randomised phase 3 trial. Lancet Oncol 11, 121-128

20. Sequist LV, Yang JC, Yamamoto $N$ et al (2013) Phase III study of afatinib or cisplatin plus pemetrexed in patients with metastatic lung adenocarcinoma with EGFR mutations. J Clin Oncol 31, 3327-3334

21. Wu YL, Zhou C, Hu CP et al (2014) Afatinib versus cisplatin plus gemcitabine for first-line treatment of Asian patients with advanced non-small-cell lung cancer harbouring EGFR mutations (LUX-Lung 6): an open-label, randomised phase 3 trial. Lancet Oncol 15, 213-222

22. Solomon BJ, Mok T, Kim DW et al (2014) First-line crizotinib versus chemotherapy in ALK-positive lung cancer. N Engl J Med 371, 2167-2177

23. Dimova NV, Hathaway NA, Lee BH et al (2012) APC/C-mediated multiple monoubiquitylation provides an alternative degradation signal for cyclin B1. Nat Cell Biol $14,168-176$

24. Kirkpatrick DS, Hathaway NA, Hanna J et al (2006) Quantitative analysis of in vitro ubiquitinated cyclin B1 reveals complex chain topology. Nat Cell Biol 8, 700-710

25. Peters JM (2002) The anaphase-promoting complex: proteolysis in mitosis and beyond. Mol Cell 9, 931-943

26. Ben-Eliezer I, Pomerantz Y, Galiani D, Nevo N and Deke $N$ (2015) Appropriate expression of Ube2C and Ube2S controls the progression of the first meiotic division. FASEB J 29, 4670-4681

27. Pan YH, Yang M, Liu LP, Wu DC, Li MY and Su SG (2018) UBE2S enhances the ubiquitination of p53 and exerts oncogenic activities in hepatocellular carcinoma. Biochem Biophys Res Commun 503, 895-902

28. Lin TH, Hsu WH, Tsai PH et al (2017) Dietary flavonoids, luteolin and quercetin, inhibit invasion of cervical cancer by reduction of UBE2S through epithelial-mesenchymal transition signaling. Food Funct 8, 1558-1568

29. Yoshimura S, Kasamatsu A, Nakashima D et al (2017) UBE2S associated with OSCC proliferation by promotion of P21 degradation via the ubiquitin-proteasome system. Biochem Biophys Res Commun 485, 820-825

30. Yang Y, Guo J, Hao $Y$ et al (2017) Silencing of karyopherin alpha2 inhibits cell growth and survival in human hepatocellular carcinoma. Oncotarget 8, 3628936304 